\title{
careful crates
}

\section{LAIDA AGUIRRE}

California State Polytechnic University, Pomona

Boxes, containers, crates can be used as a lens to look at a series of current socio-logistical phenomenon. These, presumably simple, tools of commerce have had profound impact ranging from the downturn of in-store retail to the proliferation of the return policy as a widespread approach to commerce. The box serves as the meta-design component of a large network of commodity distribution and in doing so it has created a culture of modularized thinking, a separation between product image and physical inventory and a general box-clever approach to the movement of material goods. In many ways, it has datafied our physical worlds.

'careful crates' is a mixed-media project focused on shipping/ returns as a cultural and physical contemporary condition. Referencing Richard Artschwager's 'Crates', this project serves as material resistance to a standardized bounding box economy that organizes, binds, shelters and delimits contents in anticipation of global circulation. The project consists of several props on a set where a video is filmed in collaboration with comedian Ana Fabrega. The film depicts the main character in a warehouse attempting to guess the contents of a series of boxes. 

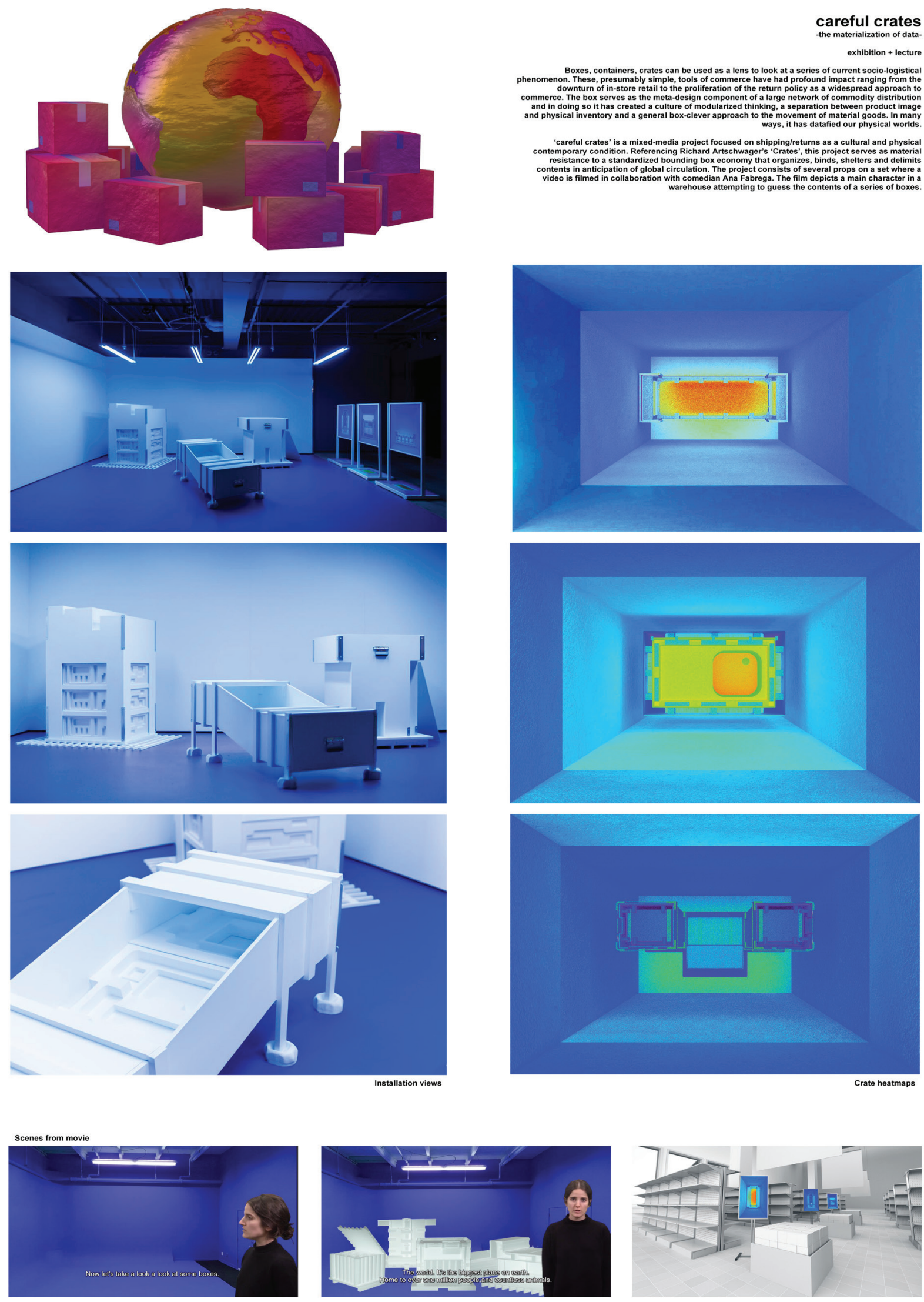